\title{
Comparison of Rate of Isolation and Characterisation of Mycobacteria by AFB Smear, LJ Medium and MGIT
}

\author{
U. Pratibha Bhat ${ }^{*}$ and Rekha Boloor ${ }^{2}$ \\ ${ }^{1}$ Department of Microbiology, K S Hegde Medical Academy, Mangaluru, India \\ ${ }^{2}$ Department of Microbiology, Father Muller Medical College, Mangaluru, India \\ *Corresponding author
}

\begin{abstract}
A B S T R A C T
Bacteriological confirmation plays a key role in the diagnosis of tuberculosis. Many systems have been developed in the recent years apart from traditional microscopy and culture on Lowenstein Jensen (LJ) medium. The objective of this study is to compare the

\begin{tabular}{|c|}
\hline Keywords \\
\hline $\begin{array}{l}\text { Tuberculosis, } \\
\text { MGIT, Diagnosis }\end{array}$ \\
\hline Article Info \\
\hline $\begin{array}{l}\text { Accepted: } \\
\text { 16 December } 2017 \\
\text { Available Online: } \\
\text { 10 January } 2018\end{array}$ \\
\hline
\end{tabular}
rate of detection of Mycobacteria by acid fast staining, LJ medium and Mycobacteria Growth Indicator Tube (MGIT). Hundred patients with clinical suspicion of pulmonary tuberculosis attending a tertiary care hospital over a period of one and a half years were enrolled in the study. Sputum samples were collected and acid fast smears were prepared. The specimens were decontaminated and cultured on LJ medium and MGIT. Growth obtained was recorded. Out of 100 suspected cases, acid fast bacilli were seen in 21 sputum samples by Ziehl-Neelsen (ZN) staining. In LJ medium culture 21 samples yielded growth and MGIT was positive in 23 samples. The sensitivity and specificity of MGIT in comparison with LJ medium culture were $100 \%$ and $97.5 \%$ respectively. MGIT culture showed faster rates of isolation compared to LJ medium with a mean detection time of 10.91days. Among the tuberculosis positive cases, 18 (78.2\%) were males and 5 (21.7\%) were females, with peak incidence (43.47\%) in age group of 31-45years. Compared to conventional methods, MGIT showed a good sensitivity and specificity and faster rates of isolation. It can be used in the diagnosis of tuberculosis as a rapid and reliable technique.
\end{abstract}

\section{Introduction}

Tuberculosis is a major health problem worldwide. It is one of the life threatening airborne infectious bacterial disease. The emergence of multidrug resistant and extremely drug resistant tuberculosis has worsened the situation (Negi et al., 2005). India is the highest tuberculosis burden country and accounts for nearly one fifth of global burden of tuberculosis (Park, 2009).
The microscopic examination of sputum to detect presence of Acid Fast bacilli (AFB) has served the world well for past 100 years and is the diagnostic basis of directly observed therapy. Reliance solely upon the limited AFB smear is one of the major factors that contribute to poor tuberculosis control in resource poor countries (Espinal et al., 2000).

The most commonly used conventional Lowenstein Jensen (LJ) medium requires 6-10 
weeks of incubation due to slow growth rate of Mycobacterium tuberculosis complex. In recent years, the development of rapid and reliable methods for culture detection of acidfast bacilli has been regarded as worthy of absolute priority. It should be such that the laboratories must be able to isolate Mycobacteria easily, quickly and accurately (Styrt et al., 1997, Chitra and Prasad, 2001). Hence, there is need to evaluate newer methods for the early detection of Mycobacterium tuberculosis complex, for effective treatment and control.

Rapid diagnosis of Mycobacterial infections is of prime importance. Meanwhile, in the developing countries, where resources are limited, patients continue to undergo diagnosis with acid-fast smears and are subsequently treated with standard anti-tuberculosis regimens. In these countries, the clinical utility of conventional antibiotic susceptibility testing methods has been offset by their long turnaround times and the utilization of more rapid methods such as BACTEC and Mycobacteria Growth Indicator Tube (MGIT) has not proven to be cost effective (Heifets and Cangelosi, 1999). Presumptive diagnosis of tuberculosis can be made on the basis of patient's history, clinical and radiological findings. But definitive diagnosis requires microscopic detection of acid fast bacilli and isolation by culture methods.

The objective of this study is to compare rate of detection of Mycobacteria by acid fast staining, LJ medium and MGIT culture methods.

\section{Materials and Methods}

This study was done at a tertiary care hospital over a period of one and a half years. 100 patients suspected of pulmonary tuberculosis based on clinical history, attending the hospital were considered.

\section{Inclusion criteria}

Patients with symptoms suggestive of pulmonary tuberculosis such as cough with expectoration of $>2$ weeks duration, fever with evening rise of temperature, night sweats, haemoptysis, weight loss, loss of appetite, chest pain were included in the study.

Patients above 16 years of age.

\section{Exclusion criteria}

Patients currently on anti-tubercular treatment Extrapulmonary tuberculosis

Patients in paediatric age group.

\section{Sample collection}

Early morning expectorated sputum samples were collected from clinically suspected cases of pulmonary tuberculosis in separate sterile, wide mouthed containers. Quality of the sample was assessed by macroscopic and microscopic examination. Watery sputum samples were excluded. Only samples appearing purulent were taken for the study.

The samples were assessed according to the Bartlett's grading system (Konemen, 1997).

\section{Sample processing}

Smears were prepared and were stained by Ziehl-Neelsen technique. The smear was graded according to RNTCP grading (Staining methods, 2000).

Samples were decontaminated and concentrated using the N-acetyl-L-CysteineSodium hydroxide (NALC-NaOH) method as recommended by the CDC's Public Health Mycobacteriology: A Guide for the Level III Laboratory. This was used to inoculate into Lowenstein Jensen medium and Mycobacteria Growth Indicator Tubes. 
Cultures on LJ medium were incubated at $37^{\circ} \mathrm{C}$ for 8 weeks. The slopes were periodically observed for the appearance of growth or contamination for a period of 8 weeks. Any growth detected was confirmed by Gram staining and acid fast staining and characterised by biochemical reactions. Also the time required for the growth to appear was noted.

MGIT Tubes with middle brook's 7H11 broth (7mL) was used (Laboratory procedure, 2005). A lyophilized vial of BBL MGIT PANTA (Polymyxin B, Amphotericin B, Nalidixic acid, Trimethoprim, Azlocillin) Antibiotic Mixture was reconstituted with $15 \mathrm{~mL}$ of BACTEC MGIT Growth Supplement. $0.8 \mathrm{~mL}$ of Growth Supplement/MGIT PANTA Antibiotic Mixture was aseptically added to the tubes. $0.5 \mathrm{~mL}$ of the concentrated specimen suspension prepared was also added. The tube was tightly recapped and mixed well. The tubes were incubated at $37^{\circ} \mathrm{C}$ for 42 days in the dark and read periodically for positivity. Positive tubes, identified by the BACTEC micro MGIT instrument were sub cultured onto LJ medium and an acid-fast smear was prepared. A Gram stained smear and culture on blood agar was also done to rule out contaminating bacteria.

\section{Characterisation of the Mycobacterial growth}

Once growth was obtained on LJ medium, each strain was characterised by the rate of growth, pigmentation and biochemical tests like niacin test, nitrate reduction test, catalase test and aryl sulfatase test.

\section{Results and Discussion}

$41 \%$ of the patients belonged to the age group of 41-60years, $33 \%$ were in the age group of $21-40$ years and $17 \%$ were in the age group of
61-80 years as shown in table 1 . The youngest patient was 17 years old and the oldest patient was 88 years old.

Out of 100 patients included in our study, 59\% of the patients were male and $41 \%$ were females. Male to female ratio was 1.4:1.

\section{ZN staining}

Out of the 100 patients, $21(21 \%)$ were found to be positive by sputum smear microscopy. Among them 8 patients(38.1\%) were of sputum grade $3+, 4(19 \%)$ were of grade $2+, 8$ $(38.1 \%)$ were of grade $1+$ and 1 patient $(4.8 \%)$ was of grade scanty as shown in table 2 .

\section{Culture on LJ medium}

$21 \%$ of the samples were positive on culture on LJ medium. The earliest detection time was 5 days and the latest detection time was 32 days. The mean detection time in LJ medium was 22.67 days with a standard deviation of 6.135 .

\section{Culture in MGIT}

$23 \%$ of the samples were positive in MGIT method. Earliest growth detected was in 4 days and the latest detection time was 20 days. The mean detection time by MGIT was 10.91 days and the standard deviation was 4.621.

Out of the 23 positive cultures 22 cultures were identified as Mycobacterium tuberculosis and 1 culture was identified as nontuberculous Mycobacteria (Mycobacterium fortuitum).

\section{Comparison of $\mathrm{ZN}$ staining with $\mathrm{LJ}$ medium culture}

Table 3 shows that, out of the 100 suspected cases, 20(95.2\%) were positive by both $\mathrm{ZN}$ staining and LJ medium culture. 1(4.8\%) 
sample negative by smear microscopy was found to be positive by culture. The sensitivity, specificity, positive predictive value of $\mathrm{ZN}$ staining compared to $\mathrm{LJ}$ medium culture was $95.2 \%, 98.7 \%$ and $95.2 \%$ respectively.

\section{Comparison of $\mathrm{ZN}$ staining with MGIT culture}

Table 4 shows that out of 100 suspected cases of pulmonary tuberculosis, 21 cases were positive by both ZN stain and MGIT culture. In addition 2 cases negative by smear microscopy were positive by MGIT. The sensitivity, specificity and positive predictive value of $\mathrm{ZN}$ stain compared to MGIT were $91.3 \%, 100 \%$ and $100 \%$ respectively.

\section{Comparison of MGIT and LJ medium culture}

Totally MGIT culture was positive in 23 cases and LJ medium culture was positive in 21 cases (as shown in table 5 and figure 1). So the rate of isolation on $\mathrm{LJ}$ medium was $91.3 \%$.

Out of the 23 strains isolated by MGIT, $2(8.7 \%)$ strains were isolated exclusively in MGIT whereas no $(0 \%)$ strains were isolated only on LJ medium. 77(97.5\%) cases were negative by both culture techniques.

The sensitivity, specificity and positive predictive value of MGIT compared to LJ medium culture were $100 \%, 97.5 \%$ and $91.3 \%$ respectively.

Fig.1 Comparison LJ medium and MGIT

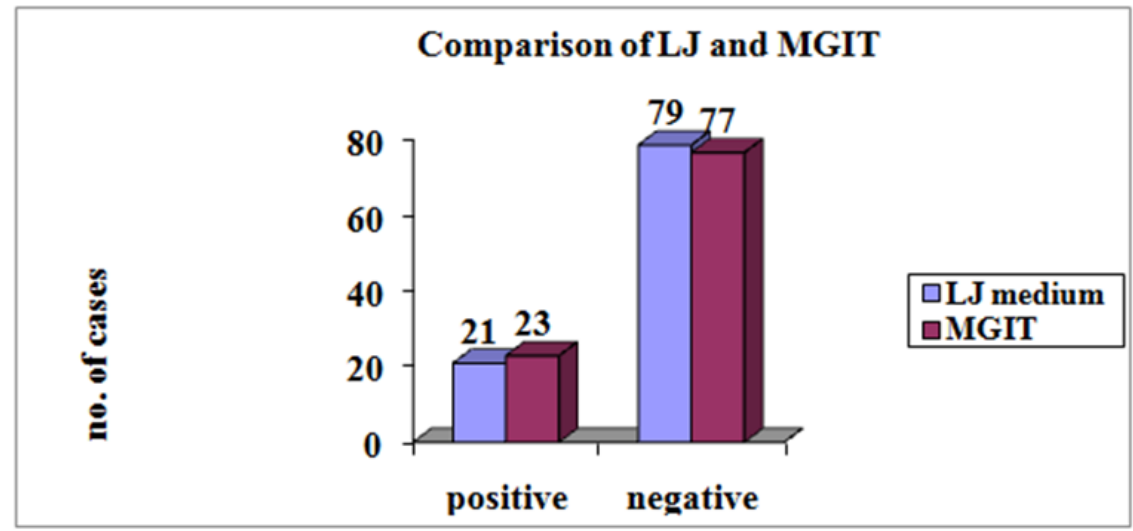

Fig.2 Duration for isolation by MGIT and LJ medium

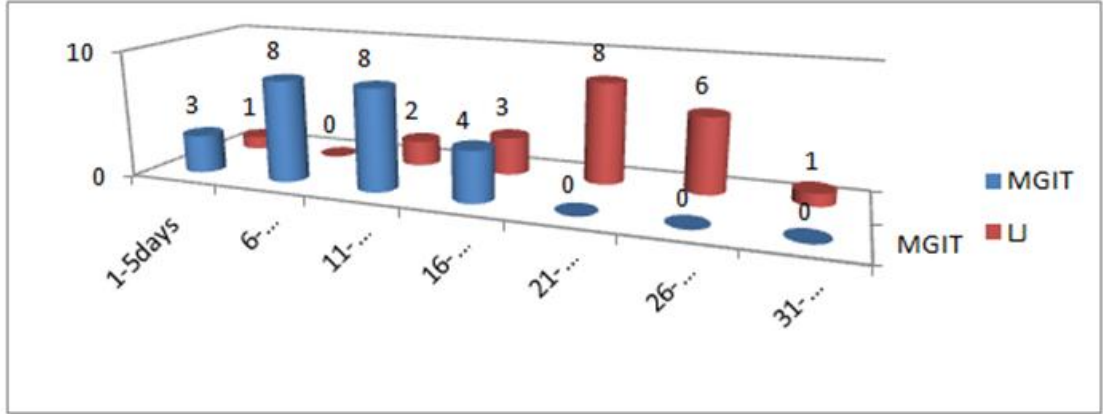


Table.1 Age distribution

\begin{tabular}{|} 
Age group(years) \\
\hline 20 and below \\
\hline $21-40$ \\
\hline $41-60$ \\
\hline $61-80$ \\
\hline Above 80 \\
\hline Total \\
\hline
\end{tabular}

\begin{tabular}{|r|}
\hline Number of patient \\
\hline 7 \\
\hline 33 \\
\hline 41 \\
\hline 17 \\
\hline 2 \\
\hline 100 \\
\hline
\end{tabular}

\begin{tabular}{|c|c|}
\hline Percentage & \\
\hline 7 \\
33 \\
41 \\
17 \\
2 \\
100 \\
\hline
\end{tabular}

Table.2 Distribution of cases according to sputum smears grading

\begin{tabular}{|c|}
\hline Sputum grade \\
\hline $3+$ \\
\hline $2+$ \\
\hline $1+$ \\
\hline Scanty \\
\hline
\end{tabular}

Number of p

patients $(\mathbf{N}=21)$
8
4
8
1

\begin{tabular}{|c|}
\hline Percentage \\
\hline $38.1 \%$ \\
\hline $19 \%$ \\
\hline $38.1 \%$ \\
\hline $4.8 \%$ \\
\hline
\end{tabular}

Table.3 Comparison of ZN staining with LJ medium culture

\begin{tabular}{|l|c|c|c|}
\hline & LJ medium positive & LJ medium negative & Total \\
\hline ZN stain positive & 20 & 1 & 21 \\
\hline ZN stain negative & 1 & 78 & 79 \\
\hline Total & 21 & 79 & 100 \\
\hline
\end{tabular}

Table.4 Comparison of ZN staining with MGIT culture

\begin{tabular}{|l|c|c|c|}
\hline & MGIT positive & MGIT negative & Total \\
\hline ZN stain positive & 21 & 0 & 21 \\
\hline ZN stain negative & 2 & 77 & 79 \\
\hline Total & 23 & 77 & 100 \\
\hline
\end{tabular}

Table.5 Comparison of MGIT and LJ medium culture

\begin{tabular}{|c|c|c|c|}
\hline & LJ medium positive & LJ medium negative & Total \\
\hline MGIT positive & 21 & 2 & 23 \\
\hline MGIT negative & 0 & 77 & 77 \\
\hline Total & 21 & 79 & 100 \\
\hline
\end{tabular}

Table.6 Duration for isolation by MGIT and LJ medium culture

\begin{tabular}{|c|}
\hline Number of days \\
\hline $1-5$ \\
\hline $6-10$ \\
\hline $11-15$ \\
\hline $16-20$ \\
\hline $21-25$ \\
\hline $26-30$ \\
\hline $31-35$ \\
\hline
\end{tabular}

\begin{tabular}{|c|c|}
\hline No. of positive MGIT & No. of positive LJ \\
\hline 3 & 1 \\
\hline 8 & 0 \\
\hline 8 & 2 \\
\hline 4 & 3 \\
\hline 0 & 8 \\
\hline 0 & 6 \\
\hline 0 & 1 \\
\hline
\end{tabular}


Table.7 Mean time to detection by MGIT in different studies

\section{Study series}

S. Rishi et al., 2007

C.Rodrigues et al., 2009

Bohy ME et al., 2009

Tortolli et al., 1999

Pfyffer GE et al.,1997

\section{Duration for isolation}

Table 6 and figure 2 show the duration for isolation by MGIT and LJ medium cultures. MGIT culture showed faster rates of isolation compared to LJ medium with mean detection time being 10.91 days.

It was also noted that among the 23 tuberculosis positive cases, peak incidence $(43.47 \%)$ was seen in age group of $31-45$ years of age. The age of the patients ranged from 18 years to 73 years. Among the 23 cases of pulmonary tuberculosis, 18 (78.2\%) were males and 5(21.7\%) were females. Male to female ratio was 3.6

The objective of our study was to find the rate of isolation of Mycobacteria by MGIT liquid culture and conventional methods. The MGIT culture method showed a faster detection rate compared to the conventional LJ medium culture technique. The mean detection time by MGIT culture was 10.91 days with a standard deviation of 4.621 .

Whereas, the mean detection time by LJ medium culture was found to be 22.67 days with standard deviation of 6.135 .

Varying mean time to detection has been observed in different studies.

Our results regarding mean time for isolation are comparable to those of Rishi et al., (2007), Tortolli et al., (1999) and Pfyffer et al., (1997).

\section{Detection time by MGIT}

\begin{tabular}{|c|}
\hline 11.23 days \\
\hline 16 days \\
\hline 18.4 days \\
\hline 13.3 days \\
\hline 9.9 days \\
\hline
\end{tabular}

Rodrigues and co-workers (2009) observed that in highly smear positive specimens (3+) a MGIT culture scored almost 99\% recovery in 48-72hrs of culture inoculation and in case of smear negative specimen an average detection time was found to be 16 days compared to 48 days by LJ method. Also, they found the system to be more advantageous in paucibacillary specimens like cerebrospinal fluid, other body fluids and fine needle aspiration cytology samples. Rishi et al., (2007) showed positivity rate of MGIT960 system alone was $34.10 \%$ and of LJ alone $1.93 \%$ and isolation rates of Mycobacteria in pulmonary and extra pulmonary samples were $61.83 \%$ and $21.98 \%$ by MGIT, $44.01 \%$ and $4.96 \%$ by LJ respectively. But for maximum recovery of Mycobacteria, a combination of MGIT960 and LJ media should be used. The use of one liquid medium and one solid medium is recommended by the Centres for Disease Control and Prevention, and nowadays the use of such a combination is acknowledged worldwide.

In our study 2 strains were isolated exclusively in MGIT. In this study, increased yield in MGIT could be partly due to bigger size of the inoculums used and partly due to addition of nutritional supplement in MGIT.

Dongsi Lu et al., (2002) showed in their study that although automated MGIT system demonstrated better sensitivity than traditional LJ slant for recovery of Mycobacteria from clinical specimens, approximately $10 \%$ of clinically significant 
Mycobacteria species would be missed by use of this system alone. MGIT system is not yet sufficiently sensitive to warrant elimination of supplemental solid medium. Bohy et al., (2009) found that MGIT tubes detected 2 isolates of Mycobacterium tuberculosis which were missed by BACTEC 460 and LJ medium, while BACTEC 460 also detected 2 isolates missed by MGIT and LJ medium. On the other hand solid media alone detected 1 isolate missed by the liquid media. Also they found that, the earliest growth of $M$. tuberculosis occurred in 15 days.

In our study, LJ medium (6 cultures) showed a higher contamination rate compared to MGIT ( 5 cultures). In the study by Tortolli et al., (1999), it was found that the BACTEC MGIT 960 system had a contamination rate of $10.0 \%$, intermediate between those of the radiometric system $(3.7 \%)$ and the egg-based medium $(17.0 \%)$. In various studies the rate of contamination depended on the concentration of sodium hydroxide, delay between specimen collection and processing due to longer specimen shipment times at some sites and different patient populations.

In our study among the 23 tuberculosis positive cases, peak incidence $(43.47 \%)$ was seen in age group of 31-45 years of age. The age of the patients ranged from 18 years to 73 years. Our study showed male preponderance among the cases of pulmonary tuberculosis. Among 23 cases, 18 (78.2\%) were males and $5(21.7 \%)$ were females. The male preponderance may be because male is more likely to come in contact with an active TB case while at work. Also male is more likely to develop habits like alcoholism and smoking.

The results of this study demonstrated that the MGIT system provided better recovery of Mycobacteria than the traditional LJ slant. However, in other studies, MGIT did not achieve $100 \%$ sensitivity for isolation of Mycobacterium tuberculosis complex. Dongsi $\mathrm{Lu}$ and co-workers (2002) found that 6\% of the strains were isolated only on LJ slants. Rishi and co-workers (2007) isolated 1.93\% of the strains on LJ alone in their study. Rodrigues and co-workers (2009) found in their study 15 of the isolates grew only on LJ medium. These results do not go along with our study, probably due to the small number of positive cultures obtained in our study.

Liquid broth cultures require 1 to 3 weeks of incubation for detection of organisms. Solid media require 3 to 8 weeks. However, solid media allow examination of colony morphology, detection of mixed cultures, and quantification of growth. Further occasional strains may grow only on solid media. For these reasons experts suggest using liquid and solid media in conjunction, with inoculation of at least one solid medium culture (Mandell, 2005). Culture still remains in-dispensable in a clinical Mycobacteriology laboratory. Rapid, sensitive and accurate detection of Mycobacterium species in clinical specimens can hasten the administration of appropriate anti mycobacterial therapy. Culture on solid medium such as LJ medium is insensitive and may take several weeks. Newer techniques which use liquid medium are able to detect Mycobacteria in less time. But they are labour intensive and have other limitations like high cost. The rapidity by which Mycobacteria are detected is the most obvious advantage of MGIT culture method. MGIT culture method is a simple, rapid and reliable method of Mycobacterial culture.

\section{References}

Bohy ME, Raafat H, Aly F, El-Aziz MA. Comparison between Mycobacteria Growth Indicator Tube (MGIT), BACTEC 460 TB System and Lowenstein-Jensen medium for detection of Mycobacterium tuberculosis. Egyptian Journal of Bronchology 2009; 
3(2):102-08.

Chitra $\mathrm{C}$ and Prasad CE. Evaluation of Mycobacteria growth indicator tube (MGIT) for primary isolation of Mycobacteria. Ind.J Tub 2001; 48:155.

Dongsi Lu, Bobbi Heeren, W. Michael Dunne. Comparison of the automated MGIT system with LJ medium for recovery of Mycobacteria from clinical specimens Am J Clin Patho 2002; 118:542-45.

Espinal MA, Kim SJ, Suarez PG, Kam KM et al., Standard short course chemotherapy for drug resistant tuberculosis: treatment outcome in 6 countries. JAMA 2000; 283:2537-45.

Fitzgerald D, Hass DW. Mycobacterium tuberculosis chap 248. In, Mandell GL (eds). Mandell, Bennet and Dolin: Principles and Practice of Infectious Diseases, $6^{\text {th }}$ edition. Churchill Livingstone, an imprint of Elsevier, 2005; 2853-85.

Heifets LB and Cangelosi GA. Drug susceptibility testing of Mycobacterium tuberculosis: a neglected problem at the turn of the century. Int J Tuberc Lung Dis 1999; 3(7):564-81.

Kent PT, Kubic GC. Public health mycobacteriology. A Guide for Level III laboratory. Atlanta: US Department of Health and Human Services, Public Health Service, Center for Disease control. Pp.1207.

Koneman E, Allen S, Janda W, Schrekenberger P, Winn W. Mycobacteria. In, Koneman E (Ed) Colour Atlas and Textbook of Diagnostic Microbiology, $6^{\text {th }}$ edition. Lippincott Williams and Wilkins Publication, 1997; 1064-1124.

Laboratory procedure. BBL MGIT- Mycobacteria Growth Indicator Tube, Revision C, 2005; 5,6 .

Negi SS, Khan SFB, Gupta S, Pasha ST et al., Comparison of conventional diagnostic modalities, BACTEC culture and polymerase chain reaction test for diagnosis of tuberculosis. Indian J Med Microbiol 2005; 23(I):29-33.

Park K. Epidemiology of Communicable Diseases Ch 5. In, Parks Textbook of Preventive and Social Medicine, $20^{\text {th }}$ edition. M/S Banarsidas Bhanot Publishers, 2009; 15975.

Pfyffer GE, Welscher HM, Kissling P, Cieslak C et al., Comparison of the Mycobacteria Growth Indicator Tube (MGIT) with Radiometric and Solid Culture for Recovery of Acid-Fast Bacilli. J Clin Microbiol 1997; 364-8.

Rishi, S., P. Sinha, B. Malhotra, N Pal. A comparative study for detection of Mycobacteria by BACTEC MGIT 960, LJ medium and direct AFB smear examination. Ind J Med Microbiol 2007; 25(4):383-6.

Rodrigues, C., S. Shenai, M. Sadani, N. Sukhadia, M. Jani, K. Ajbani, A. Sodha and A. Mehta. Evaluation of BACTEC MGIT 960 TB system for recovery and identification of Mycobacterium tuberculosis complex in a high through put tertiary care centre. Ind $J$ Med Microbiol 2009; 27(3):217-21.

Staining methods. Key steps in the staining and preparation of smears. In RNTCP at a glance. Central TB Division. Directorate General of Health services, Ministry of Health and Family Welfare 2000; 7, 8.

Styrt BA, Shinnick TM, Ridderhof JC, Crawford JT et al., Turnaround times for mycobacterial cultures. J Clin Microbiol 1997; 35:1041-42.

Tortoli E, Cichero P, Piersimoni C, Simonetti MT. Use of BACTEC MGIT 960 for Recovery of Mycobacteria from Clinical Specimens: Multicenter Study. J Clin Microbiol 1999; 37(11):3578-82.

\section{How to cite this article:}

Pratibha Bhat, U. and Rekha Boloor. 2018. Comparison of Rate of Isolation and Characterisation of Mycobacteria by AFB Smear, LJ Medium and MGIT. Int.J.Curr.Microbiol.App.Sci. 7(01): 2397-2404. doi: https://doi.org/10.20546/ijcmas.2018.701.288 